\title{
Investigating the degrading properties of three different strains of fungi on commonly used pesticides in Guyana
}

\author{
REHANA FAREED, ABDULLAH ANSARI, DIANA SEECHARRAN, LESLIE MUNROE \\ Department of Biology, University of Guyana, Georgetown, Guyana. `email: abdullah.ansari@uog.edu.gy
}

Manuscript received: 4 December 2016. Revision accepted: 28 January 2017.

\begin{abstract}
Fareed R, Ansari A, Seecharran D, Munroe L. 2017. Investigating the degrading properties of three different strains of fungi on commonly used pesticides in Guyana. Biofarmasi J Nat Prod Biochem 15: 5-14. Bioremediation is the use of microbes to remove various contaminants from the environment. The present research work was carried out from 2014 to 15. Aspergillus niger, Aspergillus flavus, and Penicillium spp. were employed for the biodegradation of pesticides commonly used in Guyana. Initially, the fungi were screened for ligninolytic potential by observing the decolorization/degradation of a synthetic dye (Remazol Brilliant blue) in PDA agar. The degradation of dyes was noted by the change in the original color of the dye and the visual disappearance of color from the fungustreated Petri plates. In addition, the accumulation of the dye by the fungal mycelium was also noticed. The fungi were then tested for their tolerance to the pesticides Diuron, Malathion, and Diazinon, respectively. Using PDA agar with amoxicillin on solid media to prevent bacterial growth. The pesticides were introduced to the fungi by way of disc diffusion. Discs of a known diameter were infused with the respective pesticides at different concentrations and placed at strategic points around the inoculated fungal mycelium. The extent of inhibition was measured by comparing the growth diameter in the sample versus the growth in the control. The pesticide diazinon was found to have inhibited the fungi more when compared to Malathion and Diuron. Finally, the fungi were used to degrade the pesticides in a liquid culture-Peptone Broth. The pesticide Diuron was selected because the fungi thrived best in all pesticide concentrations. The pesticide Diuron concentration was placed in flasks containing the peptone broth with mycelial discs of the respective fungal strain. The final concentration of the pesticide was determined by using High-Performance Liquid Chromatography (HPLC). The concentration of the pesticide in each strain of fungi was decreased. Aspergillus niger was found to have the highest rate of pesticide degradation, followed by Aspergillus flavus and Penicillium.
\end{abstract}

Keywords: Aspergillus flavus; Aspergillus niger, degradation, bioremediation, fungi, HPLC, ligninolytic potential, microbes, pesticides, Penicillium

\section{INTRODUCTION}

Environmental pollution from human activities is a major challenge faced in today's society. The industrialization development over the last five decades has resulted in an exponential increase in the production and consumption of chemicals (Coelho- Moreira et al., 2013). Consequently, improper management and disposal of these chemicals contaminate soils, groundwater, and surface waters. In addition, these chemicals have high persistence; unknown environmental pathways and their potential to bioaccumulate often result in food loss, and mutagenic and carcinogenic effects on man and other organisms within an ecosystem (Fetzner 2002; Sinha et al. 2011). Ultimately, using indigenous or introduced microorganisms to decontaminate waste sites allows for an environmentally friendly and economical solution to many hazardous pollution problems. This results in biological degradation becoming an increasingly popular alternative for treating hazardous wastes (Christian et al. 2005).

The degradation process involves converting molecules to smaller compounds that may be toxic or non-toxic or removing pesticide molecules through simple absorption or adsorption mechanism (Reece et al. 2014). Sinha et al. (2011) defined xenobiotics as compounds that are alien to a living individual and tend to accumulate in the environment. He further explained that both natural and anthropogenic activities result in the accumulation of wide ranges of toxic xenobiotic compounds in the environment. Without being metabolized by soil organisms, many xenobiotics would reach toxic concentrations. Most xenobiotics are cleared through multiple enzymes and pathways.

Fungi belonging to the genera Aspergillus and Penicillium are diverse and are classified in the family Trichocomaceae. These species are commonly found in soil, decaying organic materials, and animal feed. Trichocomaceae are saprobes and possess diverse physiological properties. Some species are opportunistic pathogens, while others are exploited in biotechnology to produce enzymes, antibiotics, and other products (Leite et al., 2012).

Organophosphates are some of the most common and toxic insecticides used today, adversely affecting humans and other organisms at low levels of exposure (Pesticide Action Network 2002). Malathion, also referred to as carbophos, maldison, and mercaptothion is a non-systemic, wide-spectrum organophosphorus insecticide used to control the household and agricultural pests (Ramadevi et al., 2012). When present in the soil, it undergoes various transformations that produce a complex pattern of metabolites. The fate of Malathion in the soil is controlled 
by the chemical, biological and physical dynamics of soil transformation activity (Ramadevi et al., 2012). Diazinon is a non-systemic organo-phosphate insecticide used on home gardens and farms to control many sucking and leaf-eating insects. It also has veterinary uses against fleas and ticks (Nyakundi et al., 2011).

Bioremediation or utilizing microorganisms to degrade toxic organo-pollutants is an efficient, economical approach that has been successful in laboratory studies. The conventional mechanisms for removing pesticides in the soil, such as chemical treatment, volatilization, and incineration, have met public opposition. This is because of the large volumes of acids and alkalis produced, the potentially toxic emissions, and the elevated economic costs (Nyakundi et al., 2011; Kumar-Praveen and Bhat, 2012). Overall, most physical and chemical cleaning technologies are expensive and rather inefficient. These clean-up methods do not suit large farms since only small soil samples are required. They are done in laboratories and hence require a lot of resources because the contaminated soil has to be excavated at a site and moved to a storage area where it can be processed. Due to environmental concerns associated with the accumulation of pesticides in food products and water supplies, there is a great need to develop safe, convenient, and economically feasible methods for pesticide remediation. For this reason, several biological techniques involving the biodegradation of organic compounds by microorganisms like bacteria and fungi have been developed (Nayakundi et al., 2011).

Coelho-Moreira et al. (2013) highlighted that diuron could be transformed abiotically via hydrolysis and photodegradation reactions, but these reactions occur at very low rates under natural conditions. Due to this, diuron is known as a potential water contaminant. Degradation products of diazinon include diazoxon and oxypyrimidine. Oxypyrimidine is the main soil and water degradation product of diazinon. Diazinon can be converted to diazoxon in the atmosphere via ultraviolet light. Diazinon released to surface waters or soil is subject to volatilization, photolysis, hydrolysis, and biodegradation (Nayakundi et al. 2011).

The transformation of diuron by $P$. chrysosporium in liquid cultures has already been documented in both stationary and shaken conditions (Coelho- Moreira et al., 2013). Singh and Singh (2010) used Aspergillus flavus as the experimental organism to degrade two textile dyes, namely Bromophenol blue and Congo red. This fungus has shown garnered positive results for dye degradation and decolorization.

This study was executed to examine microbial degradation in three strains of fungi, namely, Aspergillus niger, Penicillium spp., and Aspergillus flavus. The degradation of the dye Remazol Brilliant Blue $\mathrm{R}$ was observed in a solid medium, and pesticide degradation was done in a liquid medium. The aim of this research was to investigate the degrading properties of the three different strains of fungi on commonly used pesticides in Guyana. It focused on the following objectives: (i) To compare the ligninolytic potential of each fungal strain using Remazol Brilliant Blue dye. (ii) To compare the tolerance of each fungal strain to different concentrations of the pesticides Malathion, Diuron, and Diazinon. (iii) To determine the ability of fungi to degrade the pesticides in liquid cultures. (iv) To determine the biomass production of each fungus in liquid cultures.

\section{MATERIALS AND METHODS}

The present research work was carried out from 2014 to 15 to study the degrading properties of three fungal strains on commonly used pesticides in Guyana. The fungal cultures were obtained from the University of Guyana's laboratory. There were sub-cultured and maintained for three weeks before the commencement of the experiment. This ensured that the spores were active.

The pesticides were selected based on their availability and popularity to local farmers in Guyana. The pesticides Malathion (insecticide), Diuron (herbicide), and Diazinon (insecticide) were selected. They were then diluted to the desired concentrations required for the experiment. The Dye Remazol Brilliant blue was used, a class of anthraquinone derivative dye used in the textile industry.

\section{Preparation of agar}

The agar used was Potato Dextrose agar. This was prepared by dissolving the medium ingredients (Potato infusion, dextrose, and agar) over a heat source for approximately 15 minutes and was subsequently autoclaved for 20 minutes. The antibiotic amoxicillin was added to inhibit the growth of bacterial colonies. The dye Remazol Brilliant Blue $\mathrm{R}$ was added to the agar prepared to assess the ligninolytic potential of the fungi; this was a 1.0 $\%(w / v)$ dye solution prepared using the potato infusion.

\section{Monitoring decolorization}

Decolorizing dyes from the Petri plates was assessed by change in original color (as compared to control) and by the visual disappearance of color from the Petri plates. The radial growth of fungal mycelium and change in color was measured after a fixed interval for four days. The culture plates containing dyes were examined for the visual disappearance of color from the media of the Petri plates when compared to their respective controls.

Figure 1 shows Petri plates infused with the Remazol Brilliant Blue $\mathrm{R}$ dye and a plug of the respective fungi placed in the middle. As the fungi grow outwards, the decolorization growth diameter can be monitored. The extent of decolorization will determine the ligninolytic potential of each fungus. 


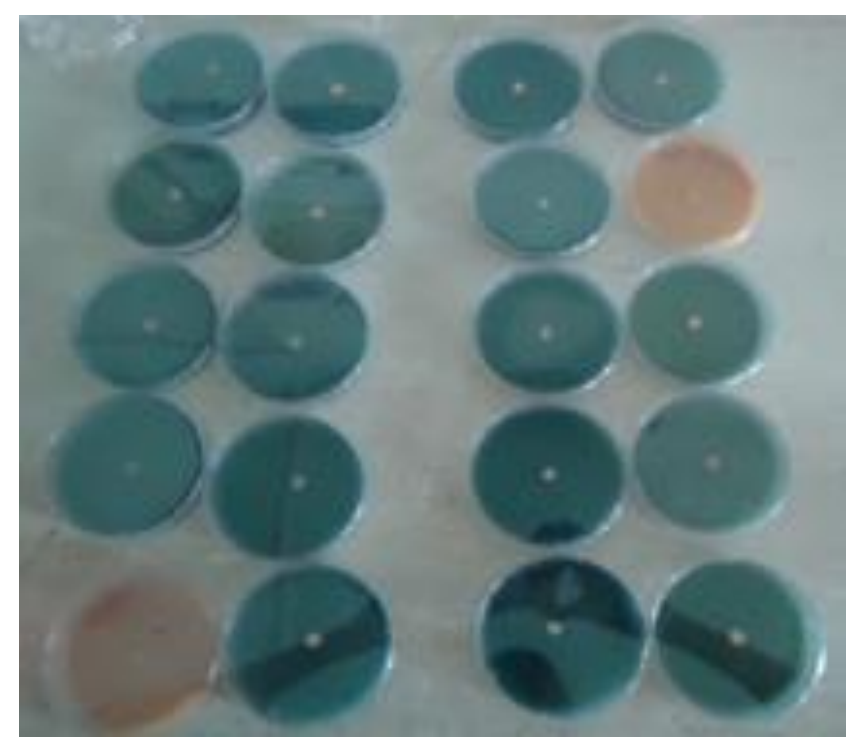

Figure 1. Infusion of Petri plates with the dye Remazol Brilliant Blue $\mathrm{R}$ and plugging of the respective fungi

Step 1-Assessing the ligninolytic potential of each strain of fungi

Approximately $30 \mathrm{ml}( \pm 1)$ of the medium was poured into each previously sterilized Petri plate, which was then allowed to cool. The Petri plates were inoculated with a plug of the respective strain and maintained at room temperature for 10 days. The decolorized zone formation was observed daily and was calculated using the formula

$\mathrm{I}=\mathrm{C}-\mathrm{T} / \mathrm{C} \mathrm{x} 100$

Where, $\mathrm{I}=$ Percentage of inhibition in fungal growth, $\mathrm{C}$ $=$ Growth in terms of colony diameter in control, and $\mathrm{T}=$ Growth in terms of colony diameter in the sample.

The plates were done in triplicates, and two types of (2) control were prepared, first with fungi only and second with dye only. The first control was used to compare the fungal growth with and without dyes. The second was used to compare the visual disappearance of color from the inoculated plates.

\section{Step 2 -Tolerance test of fungi to different concentrations of each pesticide}

The plates were inoculated with the respective fungal strains, and discs infused with the different concentrations of the pesticides were placed around the fungal inoculum. It was maintained at room temperature, and inhibition zones and zones of decolorization were observed (Figure 2 ). For each strain of the fungus, there were three plates with different concentrations of pesticides. This was done in triplicates for each strain. Three types of control were prepared, first with fungi only, second with dye only, and finally with pesticide (3) only. The first control was used to compare the fungal growth with and without dyes. The second was used to compare the visual disappearance of color from the inoculated plates, and the third was used as a reference.

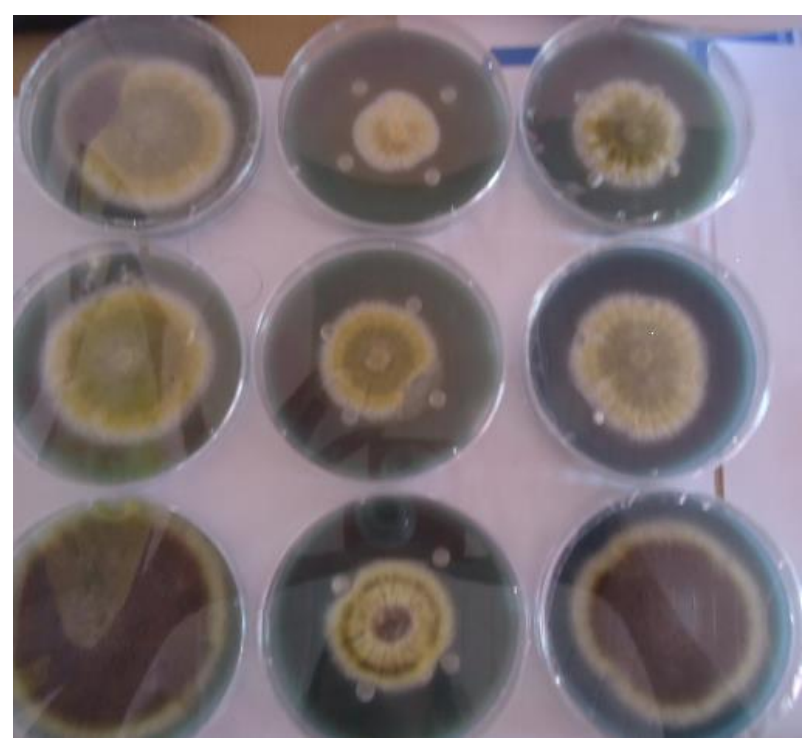

Figure 2. inhibition zones and decolorization zones

Step 3-Ability of the fungi to degrade pesticides in liquid cultures.

Agar plugs of $10 \mathrm{~mm}$ diameter were removed from an agar plate with the respective culture growing for 2 weeks. This was inoculated into $500 \mathrm{ml}$ Erlenmeyer flasks containing $60 \mathrm{ml}$ stock solution (peptone broth). A known amount/pesticide concentration was inoculated with the respective fungal strain. Each fungal strain was tested against a known concentration of the pesticide diuron. This was maintained at room temperature for seven (7) days. Each solution was then subjected to an HPLC analysis to determine the final concentration of pesticide. The fungal Biomass was collected by filtration, and the filter paper was dried for 24 hours and weighed.

\section{RESULTS AND DISCUSSION}

The rapid increase in industrialization and agricultural practices have resulted in the accumulation and contamination of many harmful chemicals in the environment. The frequency and ubiquitous use of artificial "xenobiotics" have led to immense effort to implement new technologies to purge these contaminants from the environment (Ramadevi et al. 2012). For instance, approximately thirty percent $(30 \%)$ of agricultural produce is lost to pests, thus rendering pesticides an indispensable weapon in agriculture. However, the indiscriminate use of pesticides has inflicted serious repercussions on biodiversity and human health. Bioremediation is a promising alternative that exploits microbes' natural ability to remove soil contaminants. This method is environmentally friendly, minimally hazardous, and versatile (Ramadevi et al., 2012). 
The degradation of dyes serves as an indicator of the extent of degradation that would occur when the microbes are subjected to pesticides (Ramya et al., 2007). According to Couto et al. (2001), there is a good correlation between the biodegradation of pollutants and the decolorization of polymeric dyes by ligninolytic fungi. Therefore, the decolorization of such dyes is a simple method to assess the degrading ability of the extracellular enzymes secreted by these fungi. The degrading ability of the extracellular enzymes is referred to as its ligninolytic potential. Singh and Singh (2010) highlighted that the production of extracellular enzymes such as laccase is produced by Aspergillus spp. In addition, the breakdown of most organo-pollutants by fungi is closely linked with ligninolytic metabolism. (Coelho- Moreira et al. 2013).

Aspergillus niger, Penicillium spp., and Aspergillus flavus yielded positive results for dye degradation/decolorization. This was indicated by a change and slow disappearance of the color of the dyes from the dye-containing media of the Petri plates. Additionally, a zone of different colors which is usually lighter around the fungal colony was also observed. Figure 3 shows decolorization zones observed in all strains of fungi.

This color change can be attributed to the production of extracellular enzymes by the applied fungi, which occurs during the biodegradation of the dye. Extracellular oxidases such as laccases, lignin peroxidase (Lip), and manganese peroxidase (MnP), which are involved in lignin degradation, have also been reported to decolorize dyes (Ramya et al. 2007).

In addition to the production of extracellular enzymes, absorption of the dye by the mycelium of the fungi was also observed as the mycelium of the fungi appeared blue as it grew on the surface of the dye (Knapp and Reece, 1995; Ramamurthy and Umamaheswari 2013) highlighted that absorption of dyes to the microbial cell surface is the primary mechanism of dye decolorization. This accumulation of chemicals with microbial biomass can take place on living or dead biomass. Fungal biomass is an efficient adsorbent because it contains natural polysaccharide chitin and its derivative chitosan in the cell walls. Chitosan possesses a unique molecular structure with a high affinity for many classes of dyes.

Table 1 represents the decolorization diameter of the Fungi Aspergillus niger, Penicillium, and Aspergillus flavus, respectively. The values in each table compare the growth of fungi in agar treated with the dye Remazol brilliant blue and a biotic control (without dye). For all the strains of fungus, the control exhibited higher growth rates when compared to the samples treated with the dye. This shows that the dye had some inhibitory effect on the growth of the fungi. Figure 4 shows the growth rate of each fungus in the presence of the dye Remazol Brilliant Blue compared to the growth in the control.

Comparison of the growth rate in the agar infused with the dye, Penicillium spp. recorded the highest growth rate followed by $A$. niger and then A. flavus. For days two through four, $A$. niger had the highest growth rate, followed by $A$. flavus and finally Penicillium spp.

Table 1. Decolorization diameter values and \% decolorization (Mean $\pm \mathrm{SD})$ for Aspergillus niger, Aspergillus flavus, and Penicillium

\begin{tabular}{llcc}
\hline Day & $\begin{array}{c}\text { Sample treated } \\
\text { with dye }(\mathbf{m m})\end{array}$ & Control $(\mathbf{m m})$ & $\begin{array}{c}\text { Rate of } \\
\text { decolorization } \\
(\mathbf{\%})\end{array}$ \\
\hline & & & \\
1 & Aspergillus flavus & & \\
2 & $14.00+1.73$ & $14.33+1.53$ & 2.33 \\
3 & $39.00+1.00$ & $39.33+0.58$ & 0.85 \\
4 & $55.33+2.52$ & $59.67+1.53$ & 7.26 \\
& $68.00+2.00$ & $72.33+1.53$ & 5.99 \\
& & & \\
1 & Aspergillus niger & & \\
2 & $14.67+2.52$ & $16.00 \pm 1.00$ & 8.33 \\
3 & $39.67+0.58$ & $40.33 \pm 0.58$ & 1.65 \\
4 & $65.33+2.52$ & $70.00 \pm 5.00$ & 6.67 \\
& $74.67+3.06$ & $76.33 \pm 1.53$ & 2.18 \\
& & & \\
1 & Penicillium & & 1.96 \\
2 & $16.67+1.53$ & $17.00+1.00$ & 7.44 \\
3 & $37.33+2.52$ & $40.33+0.58$ & 9.20 \\
4 & $52.67+9.45$ & $58.00+2.65$ & 9.65 \\
\hline
\end{tabular}

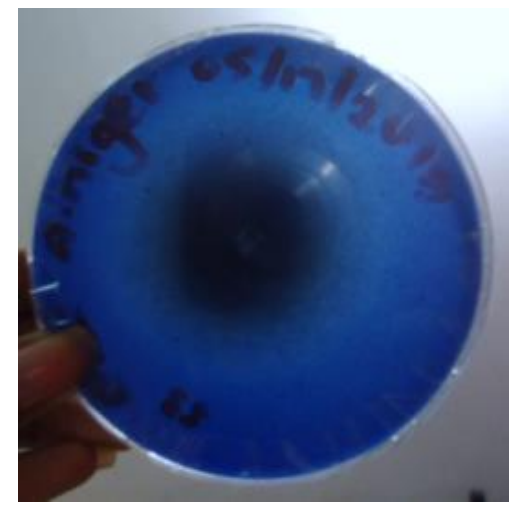

$\mathbf{A}$

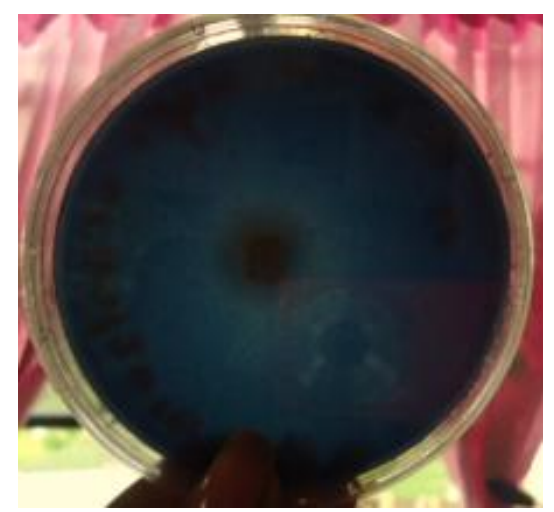

B

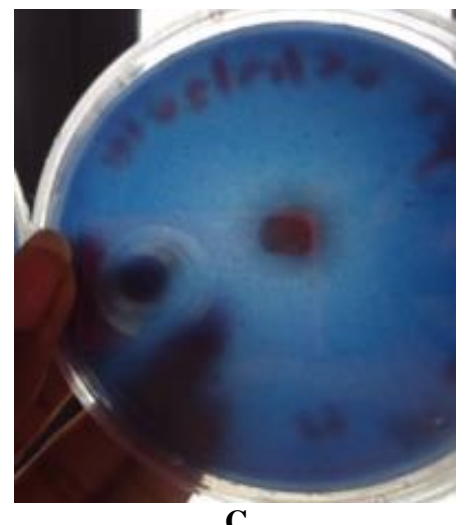

C

Figure 3. A. Aspergillus niger, B. Penicillium spp., C. Aspergillus flavus 


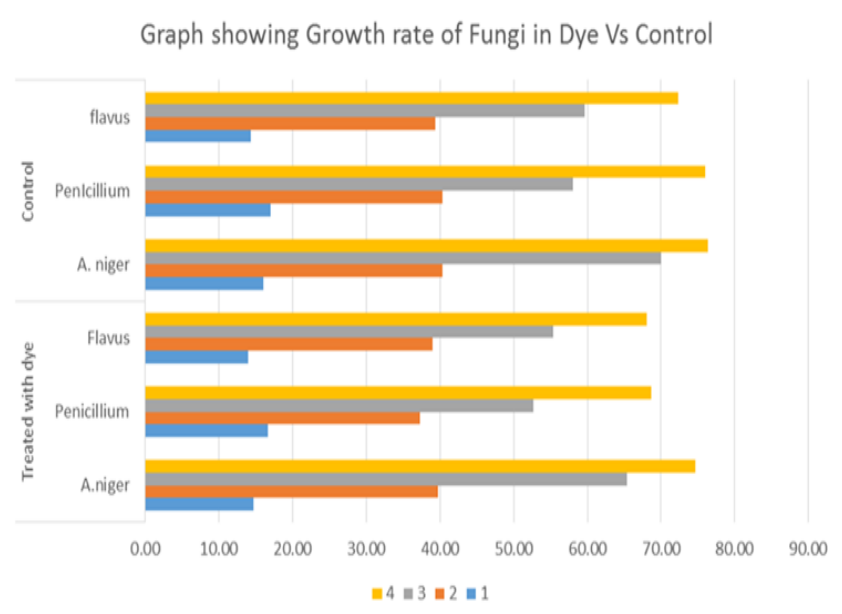

Figure 4. The growth rate of Fungi in dye vs. control

Similar patterns were observed in control with regard to day one. On day two, Penicillium spp. and A. niger recorded the same growth rate, followed by A. flavus, Day three, shows $A$. niger with the highest rate, followed by $A$. flavus. Finally, day four shows $A$. niger with the highest growth rate, followed by Penicillium and finally A. flavus.

To summarize, A. niger and Penicillium spp. recorded the highest growth rates for days one, two, and four in media treated with the dye.

When comparing the decolorization rates (\%) (Table 1), all strains of fungi exhibited varying decolorization rates. Aspergillus niger recorded the highest decolorization rate on day one, followed by Aspergillus flavus and finally Penicillium spp. For the fungi, A.niger, and A. flavus, a decrease on the second day was observed, followed by an increase in the rate on day three and, finally, a drop in the rate on the final day. However, Penicillium spp. recorded a steady increase in the rate of decolorization. Figure 5 shows average values of dye decolorization (\%) for four days to determine which strain of fungi had the highest overall ligninolytic potential. Penicillium spp. recorded the highest ligninolytic potential, followed by A.niger and finally A. flavus.

Initially, Aspergillus niger recorded the highest decolorization rate; however, as time progressed, the rate of decolorization decreased linearly. This can be attributed to the production of toxins that hindered the growth of the fungi and, consequently, the decolorization rate. As it rapidly decolorized the dye in the initial stages, the toxins produced during metabolism accumulated and hindered the production of extracellular enzymes, ultimately reducing the rate of decolorization.

Penicillium spp. recorded the highest decolorization rate, the rate increased linearly as time progressed. There was no drop in the rate of decolorization because the toxins were produced in insufficient quantities to hinder the growth of the fungi. In addition, the fungus did not decolorize the dye rapidly in its initial stages, thus limiting the effects of the amount of toxins produced. Aspergillus flavus recorded the second-highest decolorization rate; the same pattern was noticed with Penicillium, with the initial decolorization rate being low and a linear increase as time progressed.

As with Aspergillus niger, Penicillium and Aspergillus flavus also recorded a drop in decolorization rate as time progressed. However, the growth of the fungi was rapid and covered the entire Petri plate before any more observations could be recorded.

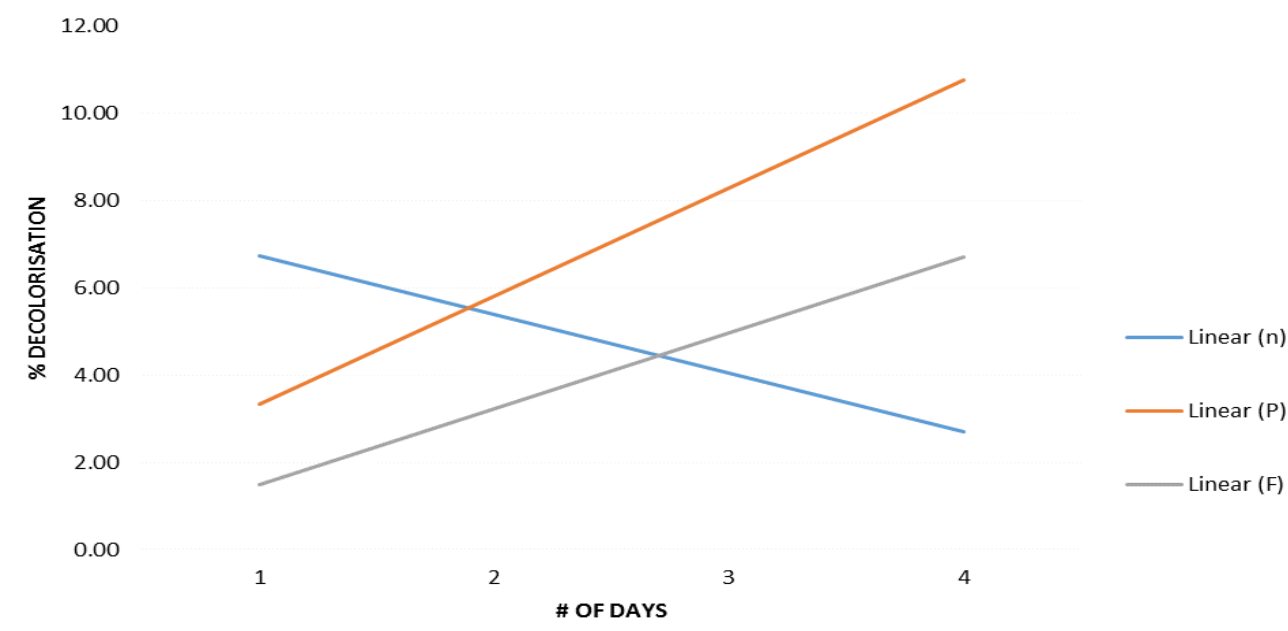

Figure 5. Rate of decolorization of each fungus ( $\mathrm{n}=$ Aspergillus niger $; \mathrm{P}=$ Penicillium and $\mathrm{F}=$ Aspergillus flavus $)$ 
It was seen that decolorization occurred by two mechanisms, mainly biosorption and enzymatic degradation. However, these reactions did not occur under optimal conditions. For instance, each fungal strain did not regulate physicochemical parameters such as media composition, $\mathrm{pH}$, and temperature. Some strains of fungi thrive best in media with glucose as the carbon source (Ramadevi et al., 2012). Additionally, nitrogen demands for growth and especially enzyme production differ markedly among fungal species. According to CoelhoMoreira et al. (2013), the production of ligninolytic enzymes with $P$. chrysosporium is much more effective under the conditions of nitrogen limitation. On the other hand, B. adusta produces more $\mathrm{LiP}$ and $\mathrm{MnP}$ in nitrogensufficient media (Pavko. 2011). Regarding pH (Knapp and Reece, 1995), the optimum $\mathrm{pH}$ depends on the medium, fungus, enzyme system, and decolorization under consideration. The majority of researchers suggest that the optimum $\mathrm{pH}$ values are likely to be in the range of 4-4.5. The optimal decolorization temperature for a particular process has to be selected from case to case according to the mentioned parameters (Singh and Singh 2010).

Hussaini et al. (2013) defined pesticides as any substance or a mixture of substances that are used to control destructive pests such as insects, plant disease organisms, and weeds, including many other living organisms such as nematodes, arthropods other than insects, and vertebrates that endanger our food supply, health, or comfort. The term pesticide can also refer to chemical substances that alter the biological processes of living organisms (Timchalk 2001).

The application of pesticides may adversely affect the different life forms within ecosystems. Coelho-Moreira et al. (2013) elucidated that approximately 90\% of agricultural pesticide applications never reach their target organisms; instead, they are dispersed through the air, soil, and water.
Ramadevi et al. (2012) highlighted that Aspergillus spp. is an effective biodegrading organism and can be effectively used to create bioremediation systems in malathion-contaminated soil. It was noted that due to their high biodegradation activity, this fungal strain has the potential to be used as biological agents for the remediation of soil, water, or crops contaminated with pesticide malathion (Ramadevi et al. 2012). Leitao (2009) explained that penicillium spp. has demonstrated their ability to degrade different xenobiotic compounds with low cosubstrate requirements and could be potentially interesting for developing economically feasible processes for pollutant transformation. Furthermore, Penicillium strains can live in saline environments, an advantage of these microorganisms over the others in the bioremediation field (Leitao. 2009).

The three strains of fungi were placed in solid media with different concentrations of the pesticides Diuron, Malathion, and Diazinon. The fungi growth diameter and decolorization were measured and observed during growth. Aspergillus niger recorded the highest average growth rate in all concentrations for each pesticide except for $10 \%$ Diazinon and the recommended dosage (Table 2). Penicillium spp. was second, followed by Aspergillus flavus. However, the percent inhibition values (Table 3) show that Aspergillus niger had the highest inhibition percent in Diuron at $1 \%$, Malathion at $1 \%$, and for all concentrations of Diazinon.

The percent inhibition values take the growth of the fungi in the sample and express it as a percent of the growth in the control. Hence the extent of inhibition of each pesticide and concentration can be calculated. Figures 6-7 are observed inhibition zones and each fungus's growth diameter. These were formed initially as the fungal mycelia grew outwards, occupying the plate. However, these inhibition zones disappeared after three days, most likely because the fungi acclimatized to the physiochemical changes in the media due to the pesticides.

Table 2. Average growth diameter (mm) for each fungus in different concentrations of each pesticide for 4 days.

\begin{tabular}{|c|c|c|c|c|c|c|c|c|c|}
\hline \multirow{2}{*}{ Fungal strain } & \multicolumn{3}{|c|}{ Diuron } & \multicolumn{3}{|c|}{ Malathion } & \multicolumn{3}{|c|}{ Diazinon } \\
\hline & $1 \%$ & $10 \%$ & R.D & $1 \%$ & $10 \%$ & R.D & $1 \%$ & $10 \%$ & R.D \\
\hline A. flavus & 28.14 & 35.42 & 28.06 & 38.625 & 35.08 & 34.67 & 35.83 & 35.33 & 37.67 \\
\hline A. niger & 43 & 44.67 & 43.92 & 44.67 & 46.33 & 43.67 & 38.50 & 25.67 & 33.58 \\
\hline Penicillium spp. & 38.92 & 36.63 & 37.75 & 40.625 & 37.25 & 34.96 & 36.58 & 25.50 & 32.17 \\
\hline
\end{tabular}

Table 3. Percent (\%) inhibition of each fungus in different concentrations of each pesticide for four days

\begin{tabular}{|c|c|c|c|c|c|c|c|c|c|}
\hline \multirow{2}{*}{ Fungal strain } & \multicolumn{3}{|c|}{ Diuron } & \multicolumn{3}{|c|}{ Malathion } & \multicolumn{3}{|c|}{ Diazinon } \\
\hline & $1 \%$ & $10 \%$ & R.D & $1 \%$ & $10 \%$ & R.D & $1 \%$ & $10 \%$ & R.D \\
\hline A. flavus & 10.23 & 13.97 & 8.02 & 4.06 & 12.84 & 20.05 & 13.39 & 12.97 & 9.98 \\
\hline A. niger & 12.81 & 8.70 & 9.04 & 10.85 & 5.55 & 11.37 & 30.92 & 46.48 & 40.54 \\
\hline Penicillium spp. & 8.80 & 13.96 & 12.58 & 3.84 & 13.23 & 20.02 & 16.93 & 41.48 & 26.52 \\
\hline
\end{tabular}



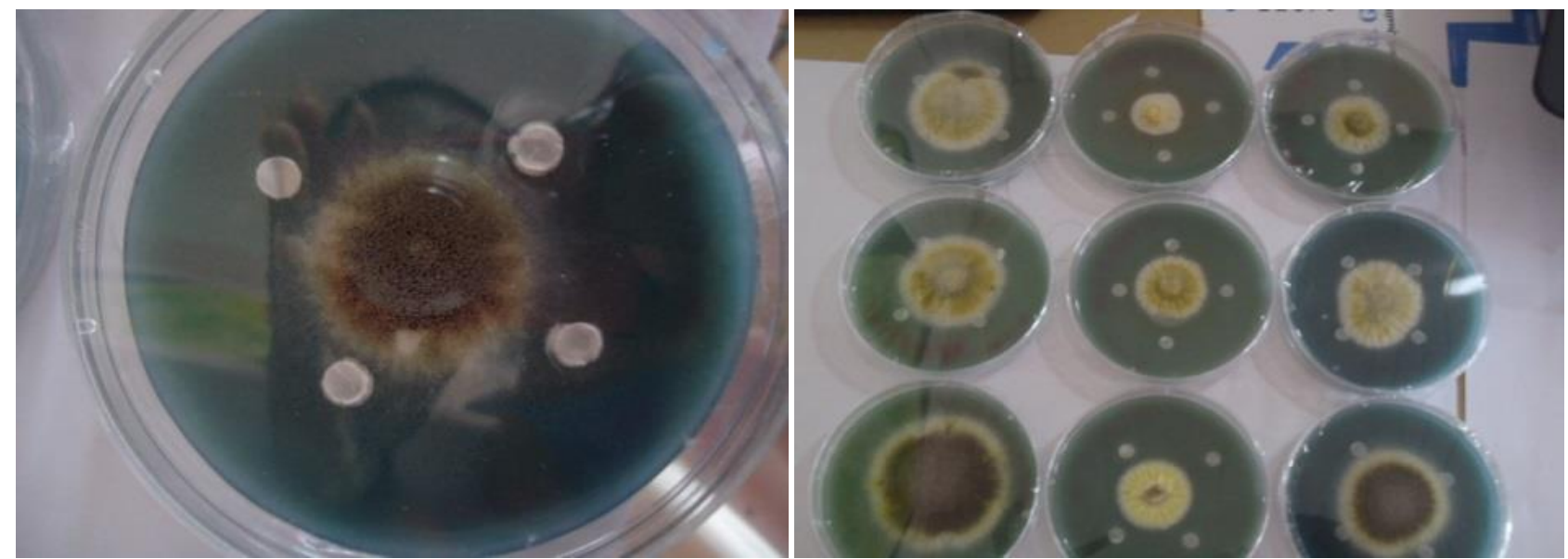

Figure 6. Inhibition zones

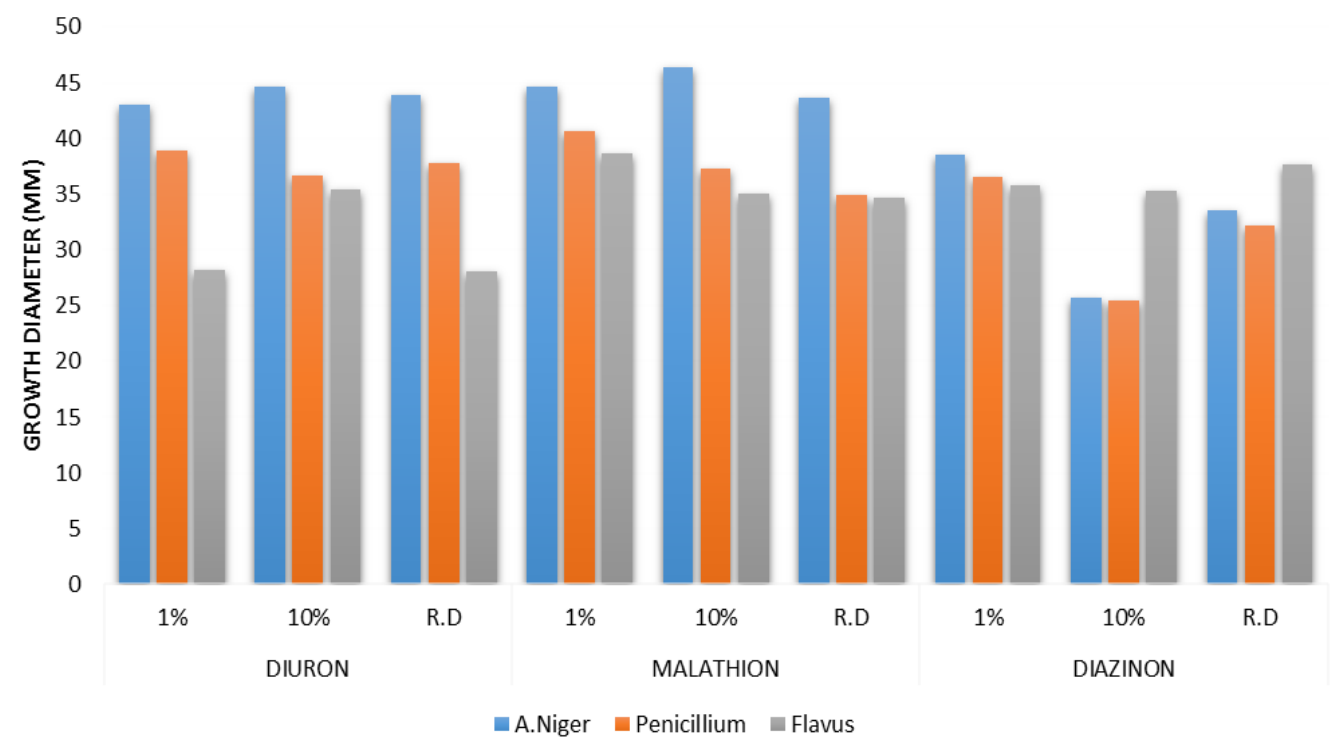

Figure 7. Growth diameter of each fungus in the different concentrations of the pesticides Diuron, Malathion, and Diazinon

When comparing the fungal growth (Table 2), the fungi thrived best in Diuron and Malathion's pesticides. However, decolorization was observed in all plates treated with the three pesticides.

Aspergillus niger grew best in all concentrations of pesticides except for $10 \%$ diazinon, followed by Penicillium in all concentrations except $10 \%$ diazinon. Aspergillus flavus had the lowest growth rate in all pesticides except Diazinon $10 \%$ and the recommended dosage. Even though inhibition was present in the plates treated with pesticides, decolorization was observed in all the plates. They grew outwards and over the discs infused with various concentrations of pesticides. This shows that the pesticides did not affect the fungal strains' production and secretion of extracellular enzymes.

The ANOVA tests revealed significant differences in the inhibition rate in Diuron $1 \%$ and for Malathion and Diazinon recommended dosages. No significant differences were noticed between fungi except in the pesticide Diazinon (not shown).

After the initial screening process, which involved assessing the ligninolytic potential and pesticide tolerance, the fungal strains were allowed to grow in liquid cultures. The only carbon source available was from the nutrient broth and a known concentration of Diuron (CoelhoMoreira et al., 2013). Diuron is a phenyl urea herbicide applied to various crops, especially sugar cane. The compound acts in photosynthetic organisms by blocking electron transport. The initial concentration was $30 \mathrm{ppm}$ after the High-Pressure liquid chromatography (HPLC) analysis was conducted.

The respective fungal strain was inoculated with the pesticide and nutrient broth. After the fungal biomass was collected, the filtrate was analyzed using HPLC, and the final concentration of the pesticide subjected to each fungal strain was determined. The final concentration of Diuron was determined by using HPLC analysis (Figure 8). 


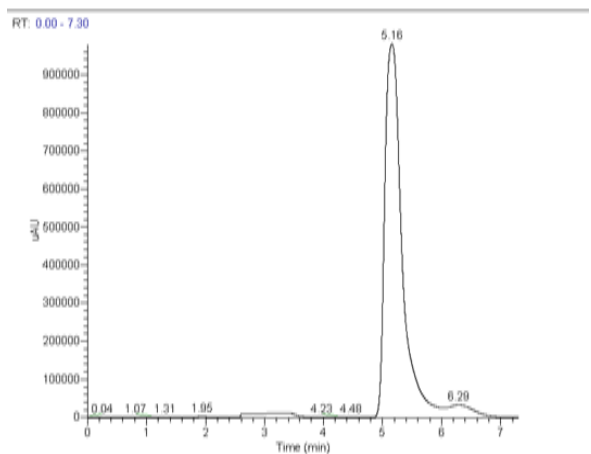

A

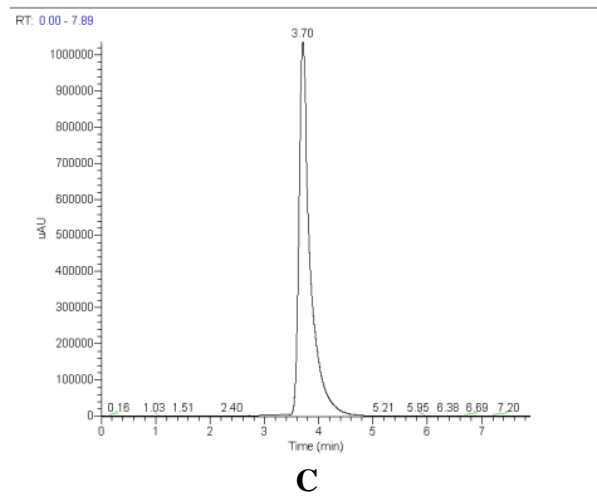

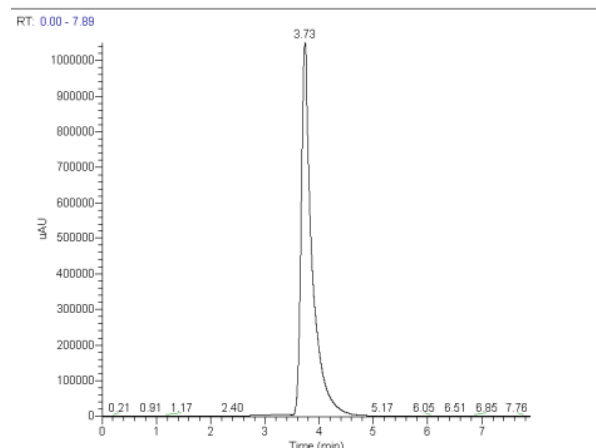

B

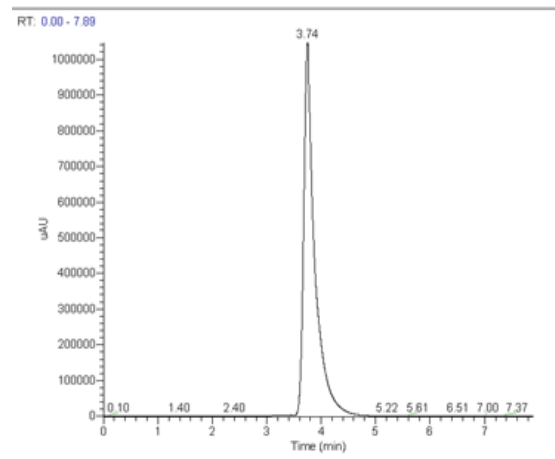

D

Figures 8. A. Retention time of the initial (pure) pesticide sample before it was treated with each fungus. B. After A. flavus, C. After A. niger, D. After Penicillium spp.

The retention time is the amount of time it takes for the compound (Diuron) to travel through the column during separation to the detector. The retention time is assigned to a corresponding peak, indicating that the interest compound is being detected. By comparing retention times, the difference in concentration can be seen by noting the decrease in retention times and the area under the peak. The retention time peaks were done in duplicates and were observed at 5.16 and 5.15 minutes, respectively. This correlates with retention times of Diuron reported in the literature, which is usually around 5-6 minutes.

Figure 9 depicts the retention time peaks of the pesticide after being treated with each of the respective fungi. Compared to the control sample's retention time peak, the time recorded after being treated with the fungi was less. The retention time peaks for the sample treated with the fungi all peaked around three minutes. In addition, the area under the curve was larger for the control sample compared to the samples treated with fungi. Aspergillus niger recorded the fastest retention time, followed by Aspergillus flavus and then Penicillium spp. The graphs produced from the HPLC analysis provided insight into Pesticide degradation, as the decreased retention times and reduced area under the curve suggest the pesticide concentration decreased. By using data garnered from these graphs, values of the concentration of Diuron in Parts per million (PPM) were determined (Table 4).

It was evident that degradation occurred because the concentration decreased in all the samples treated with fungi. The concentration of the control was still found to be the same as initially recorded.

The fungus Penicillium spp. recorded the highest pesticide concentration of $26.77 \mathrm{ppm}$, followed by Aspergillus flavus with $26.63 \mathrm{Ppm}$ and finally Aspergillus niger with $26.60 \mathrm{ppm}$. It can be seen that Aspergillus niger was most effective in degrading the pesticide Diuron as it recorded the lowest concentration of pesticide. Concerning biomass production in the pesticide Diuron, Aspergillus niger had the highest production, followed by Aspergillus flavus and, finally, Penicillium (Table 5).

Table 4. Percentage degradation (MEAN \pm SD) of each strain of fungus on the pesticide Diuron

\begin{tabular}{lccc}
\hline $\begin{array}{c}\text { Fungal } \\
\text { strain }\end{array}$ & $\begin{array}{c}\text { Initial } \\
\text { concentration } \\
\text { of pesticide } \\
\text { (ppm) }\end{array}$ & $\begin{array}{c}\text { Final } \\
\text { concentration } \\
\text { of pesticide } \\
\text { (ppm) }\end{array}$ & $\begin{array}{c}\text { \% } \\
\text { Degradation }\end{array}$ \\
\hline A. flavus & $30.709 \pm 0.077$ & $26.630 \pm 0.081$ & 86.718 \\
A. niger & $30.709 \pm 0.077$ & $26.601 \pm 0.057$ & 86.625 \\
Penicillium & $30.709 \pm 0.077$ & $26.770 \pm 0.043$ & 87.176 \\
\hline
\end{tabular}

Table 5. Biomass production (MEAN + SD) of each strain of fungi in the pesticide Diuron

\begin{tabular}{lll}
\hline \multicolumn{1}{c}{ Fungal strain } & $\begin{array}{c}\text { Sample treated } \\
\text { with pesticide }(\mathbf{g})\end{array}$ & \multicolumn{1}{c}{ Control (g) } \\
\hline A. flavus & $0.38 \pm 0.01$ & $0.38 \pm 0.01$ \\
A. niger & $0.40 \pm 0.01$ & $0.41 \pm 0$ \\
Penicillium & $0.38 \pm 0.01$ & $0.39 \pm 0.01$ \\
\hline
\end{tabular}




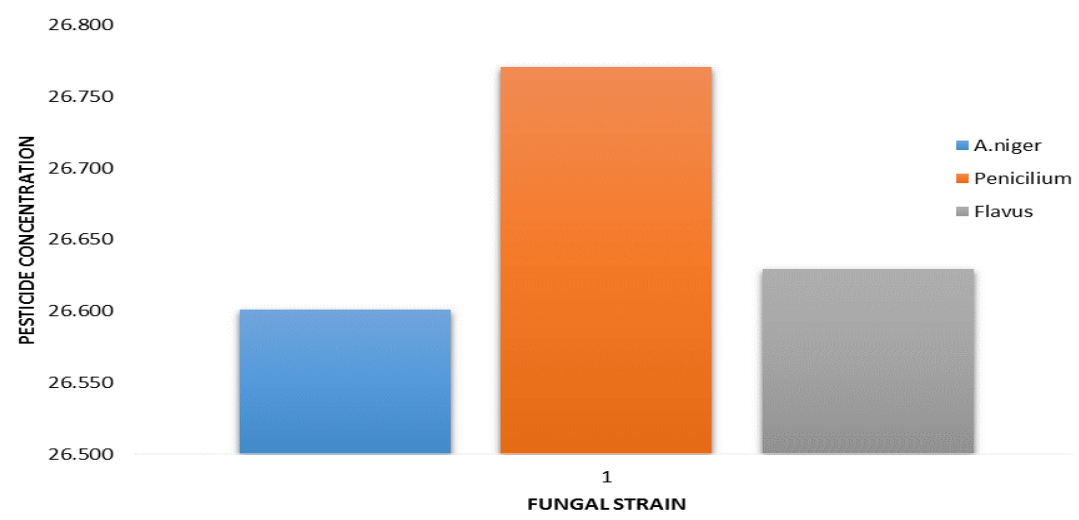

Figure 9. The final concentration of pesticide after being treated with each fungus

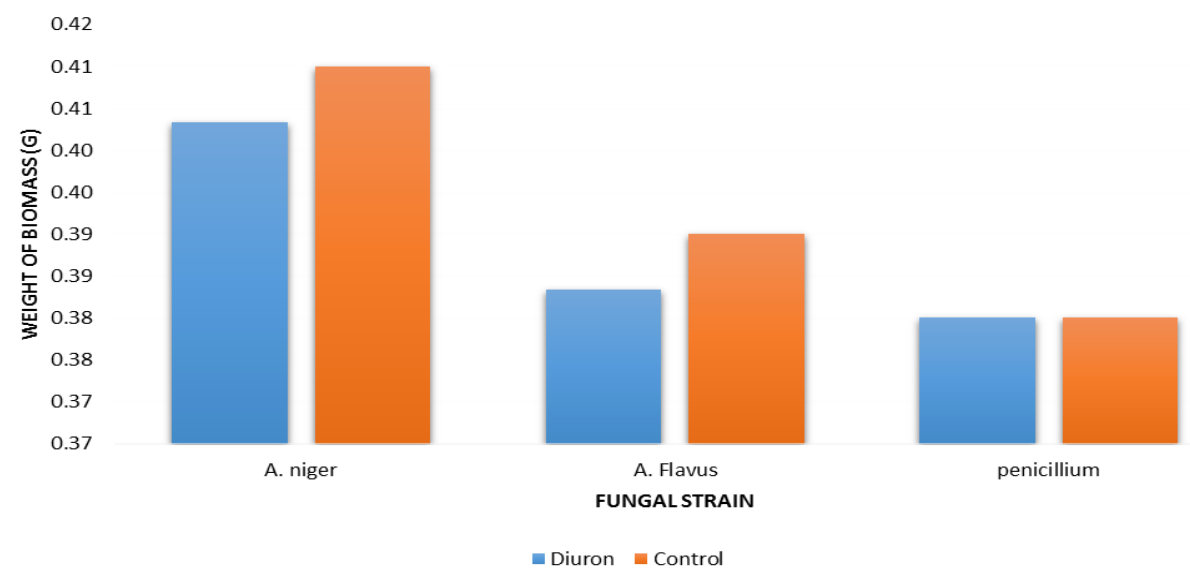

Figure 10. Biomass production in pesticide vs. control

However, biomass production in the controls for Aspergillus niger and Aspergillus flavus were higher when compared to the production in the pesticide-treated sample. This shows that the pesticide affected/inhibited the biomass production of the fungi. However, this was not observed in Penicillium species which shows that biomass production is the same in control and pesticide. This might be due to increased tolerance of the Penicillium spp. to the pesticide. Additionally, the fungi Aspergillus niger and Aspergillus flavus thrived better in the liquid media with the pesticide and in control than Penicillium spp. Figure 10 represents these observations.

Additionally, it was observed that there is a relationship between biomass production and pesticide degradation. For instance, Aspergillus niger recorded the highest degradation and highest biomass production. Similar trends were noticed for Aspergillus flavus and Penicillium spp. However, this is expected since more growth/spore production would utilize more of the pesticide as a carbon source, reducing the pesticide concentration.
In conclusion, mycoremediation is an environmentally friendly alternative that can be used to clean up toxins in the environment. This study found that the fungal strains; Aspergillus niger, Penicillium spp., and Aspergillus Flavus all exhibited ligninolytic potential as they all decolorized the textile dye remazol brilliant blue. The strain Penicillium was shown to have the highest decolorization rate, followed by Aspergillus flavus and Aspergillus niger. The three strains of fungi were more tolerant to the pesticides Malathion and Diuron as it was observed that Diazinon had the highest inhibition percent in all strains of the fungi tested. Concerning pesticide degrading properties, Aspergillus niger recorded the highest rate, followed by Aspergillus flavus and Penicillium spp.

\section{REFERENCES}

Christian V, Shrivastava R, Shukla D, Modi HA, Vyas BR. 2005. Degradation of xenobiotic compounds by lignin-degrading white-rot 
fungi: enzymology and mechanisms involved. Indian J Exp Biol 43 (4): 301-302.

Coelho-Moreira JDS, Bracht A, Souza ACDSD, Oliveira RF, SáNakanishi ABD, Souza CGMD, Peralta RM. 2013. Degradation of diuron by Phanerochaete chrysosporium: role of ligninolytic enzymes and cytochrome P450. BioMed Res Intl 2013. DOI: $10.1155 / 2013 / 251354$

Couto SR, Domínguez A, Sanroman A. 2001. Utilisation of lignocellulosic wastes for lignin peroxidase production by semi-solidstate cultures of Phanerochaete chrysosporium. Biodegradation 12 (5): 283-289.

Fetzner S. 2002. Biodegradation of Xenobiotics. In: Doelle, Da Silva (eds.). Biotechnology. In: Encyclopedia of Life Support Systems (EOLSS), developed under the Auspices of the UNESCO, Eolss Publishers, Oxford, UK.

Hussaini SZ, Shaker M, Iqbal MA. 2013. Isolation of bacterial for degradation of selected pesticides. Advances in Bioresearch 4 (3): 82 85.

Knapp JS, Newby PS, Reece LP. 1995. Decolorization of dyes by wood rotting basidiomycete fungi. Enzyme Microb Technol 17 (7): 664 668.

Kumar-Praveen GN, Bhat SK. 2012. Fungal Degradation of Azo dye-Red 3BN and Optimization of Physico-Chemical Parameters. ISCA J Biol Sci 1 (2): 17-24

Leitão AL. 2009. Potential of Penicillium species in the bioremediation field. Intl J Environ Res Public Health 6 (4): 1393-1417.

Leite D, Yamamoto A, Amadio J, Martins E, Do Santos F. 2012 Trichocomaceae: Biodiversity of Aspergillus species and Penicillium species in libraries. J Infect Dev Ctries 6 (10): 734-743.
Nyakundi W, Magoma G, Ochora J, Nyende A. 2011. Biodegradation of Diazinon and Methomyl. J Appl Technol Environ 1 (2): 107-124.

Pavko A. 2011. Fungal decolorization and degradation of synthetic Dyes; Some chemical engineering aspects. InTech, Croatia.

Pesticide Action Network. 2002. PAN North America. Organophosphates: http://www.panna.org/resources/organophosphates. Retrieved January 15,2015

Ramadevi C, Nath MM, Prasad MG. 2012. Mycodegradation of malathion by a soil fungal isolate, Aspergillus niger. Intl J Basic Appl Chem Sci 2 (1): 108-115.

Ramamurthy V, Umamaheswari G. 2013. Biodegradation of synthetic dyes by Aspergillus terreus inoculated on solid media. Intl J Innov Res Sci 2 (12): 7821-7827.

Ramya M, Anusha B, Kalavathy S, Devilakshi S. 2007. Biodecolorization and biodegradation of reactive blue by Aspergillus spp. African J Biotechnol 6 (12): 1441-1445.

Reece JB, Urry LA, Caik ML, et al. 2014. Campbell Biology. 10th ed. Benjamin Cummings, New York.

Singh L, Singh VP. 2010. Biodegradation of textile dyes, bromophenol blue and congo red by fungus Aspergillus flavus. Intl J Sci Technol 5: 235-242.

Sinha S, Chattopadhyay P, Pan I, Chatterjee S, Chanda P, Bandyopadhyay D, Sen SK. 2011. Microbial transformation of xenobiotics for environmental bioremediation. African J Biotechnol 8 (22): 60166027.

Timchalk C. 2001. Organophosphate Pharmacokinetics. In: Krieger R (ed.). Handbook of Pesticide Toxicology, 2nd ed. Academic Press, San Diego. 